CRYSTALLOGRAPHIC COMMUNICATIONS

ISSN 2056-9890

Received 3 August 2020

Accepted 3 August 2020

Edited by A. V. Yatsenko, Moscow State University, Russia

Keywords: crystal structure; nitroflavone; anticancer agent; $\pi$ - $\pi$ - interactions; DFT calculations.

CCDC reference: 2021153

Supporting information: this article has supporting information at journals.iucr.org/e

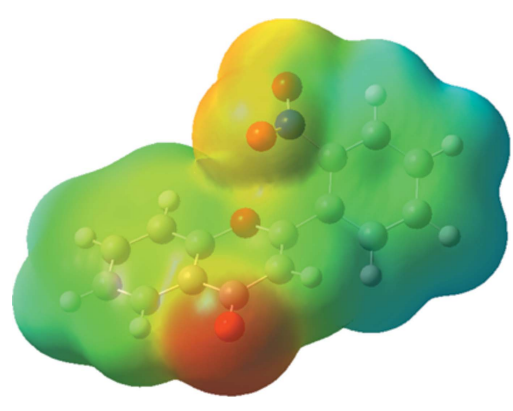

\section{Structural (at $100 \mathrm{~K}$ ) and DFT studies of 2 '-nitro- flavone}

\author{
Evgenii Oskolkov, ${ }^{a *}$ Tatiana Kornilova, ${ }^{a}$ Preciosa America Chavez, ${ }^{a}$ John P. \\ Tillotson $^{\mathbf{b}}$ and Tatiana V. Timofeeva ${ }^{\mathbf{a}}$
}

\begin{abstract}
${ }^{\mathbf{a}}$ Department of Chemistry, New Mexico Highlands University, Las Vegas, New Mexico, 87701, USA, and ${ }^{\mathbf{b}}$ School of Chemistry and Biochemistry, Georgia Institute of Technology, Atlanta, Georgia, 30332, USA. *Correspondence e-mail: zhekic@gmail.com
\end{abstract}

The geometry of the title molecule [systematic name: 2-(2-nitrophenyl)- $4 \mathrm{H}$ chromen-4-one], $\mathrm{C}_{15} \mathrm{H}_{9} \mathrm{NO}_{4}$, is determined by two dihedral angles formed by the mean plane of phenyl ring with the mean planes of chromone moiety and nitro group, being $50.73(5)$ and $30.89(7)^{\circ}$, respectively. The crystal packing is determined by $\pi-\pi$ interactions and $\mathrm{C}-\mathrm{H} \cdots \mathrm{O}$ contacts. The results of DFT calculations at the B3LYP/6-31G* level of theory provided an explanation of the unusually large dihedral angle between the chromone moiety and the phenyl group. The electrostatic potential map on the molecular surface was calculated in order to determine the potential binding sites to receptors.

\section{Chemical context}

The naturally occurring group of heterocyclic compounds known as flavonoids has received considerable attention over the past 15 years. The synthesis and applications of flavones and their derivatives have been studied extensively because of their diverse pharmaceutical properties. Besides their physiological role in plants (Agati et al., 2012), this class of compounds has demonstrated antiallergic, antiviral, anxiolytic and anti-inflammatory activities (Manthey et al., 2001). Several synthetic flavonoids and their nitro derivatives, including a few halogen-substituted compounds, have been found to act as highly competitive ligands for benzodiazepine receptors, suggesting a possible use as anxiolytic drugs (Marder et al., 1995). Most importantly, several nitro derivatives of flavones have been reported to possess antiproliferative properties against human and murine cancerous cells, by the mechanism of induced apoptosis (Blank et al., 2004). Moreover, some flavonoids have been found to be capable of restoring the viability of human vascular endothelial cells, thus providing both cytoprotective and cytotoxic effects on normal and cancerous cells, respectively (Ramos, 2008). The title compound, 2'-nitroflavone, has previously been shown to effectively inhibit human and murine tumor cell activity without affecting the non-tumor cells. Induced apoptosis molecular mechanisms have been studied in vitro for HeLa human cervix carcinoma (Cárdenas et al., 2008) and in vivo in murine adenocarcinoma cells (Cárdenas et al., 2009). Several haematological cancer cell lines were used in the cytotoxicity evaluation of the title compound, along with a culture of healthy peripheral blood mononuclear cells (PBMCs); the $\mathrm{IC}_{50}$ values (drug concentrations needed to induce a $50 \%$ inhibition of cell growth) after 2'-nitroflavone treatment 
ranged from $1 \pm 0.5 \mu \mathrm{mol} \mathrm{L}^{-1}$ to $9 \pm 1.4 \mu \mathrm{mol} / \mathrm{L}$ for various neoplastic cells, while the healthy cells $\mathrm{IC}_{50}$ was found to be over $80 \mu \mathrm{mol} \mathrm{L}{ }^{-1}$, effectively leaving the cells intact under the concentrations sufficient for cancerous cells (Cárdenas et al., 2012). Despite the evident importance of nitroflavone derivatives, structural studies until now have been limited to only one reported nitroflavone-based compound (Kendi et al., 1996). In this work, a combined study consisting of X-ray diffraction (XRD) structural analysis and quantum-chemical DFT calculations was carried out in order to obtain insight into the structure-property relationship, and more specifically the effect of the nitro substituent in the ortho-position of the phenyl moiety of a flavone.<smiles>O=c1cc(-c2ccccc2[N+](=O)[O-])oc2ccccc12</smiles>

\section{Structural commentary}

The molecular structure of $2^{\prime}$-nitroflavone is presented in Fig. 1. The mean plane of the benzene ring makes dihedral angles of $50.73(5)$ and $30.89(7)^{\circ}$ with the mean planes of the chromone moiety and the nitro group, respectively. The dihedral angle between mean planes of the chromone and benzene groups is unusually large when compared to other ortho-substituted flavone derivatives. For instance, the mol-

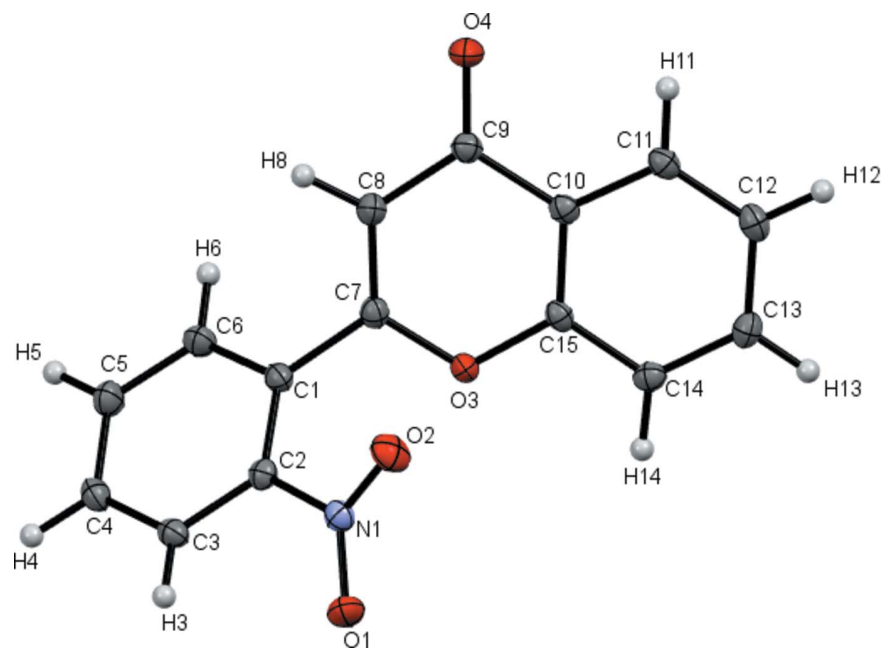

Figure 1

A view of the molecular structure of the title compound with the atomlabeling scheme. Displacement ellipsoids are drawn at the $50 \%$ probability level.
Table 1

Hydrogen-bond geometry $\left(\AA{ }^{\circ}\right)$.

\begin{tabular}{lllll}
\hline$D-\mathrm{H} \cdots A$ & $D-\mathrm{H}$ & $\mathrm{H} \cdots A$ & $D \cdots A$ & $D-\mathrm{H} \cdots A$ \\
\hline $\mathrm{C} 3-\mathrm{H} 3 \cdots \mathrm{O} 4^{\mathrm{i}}$ & $0.933(17)$ & $2.675(16)$ & $3.198(3)$ & $116.1(12)$ \\
$\mathrm{C} 4-\mathrm{H} 4 \cdots \mathrm{O} 4^{\mathrm{i}}$ & $0.971(16)$ & $2.446(16)$ & $3.109(3)$ & $125.3(12)$ \\
\hline
\end{tabular}

Symmetry code: (i) $-x+1, y+\frac{1}{2},-z+\frac{5}{2}$.

ecule of 2 -methoxyflavone was reported to be almost planar, with a dihedral angle of $2.9^{\circ}$ (Wallet et al., 1990). Even in the flavonoid with a bulky carbazole substituent in the same position, this dihedral angle is only $29.2^{\circ}$ (Zheng, 2018). The length of the single bond between the chromone and benzene moieties is 1.469 (2) $\AA$, indicating some $\pi-\pi$ conjugation. The unusually large dihedral angle in the title molecule can be attributed to the steric tension between the nitro group and the chromone oxygen atom, whereas in the carbazole derivative this substituent is twisted far enough from the plane of the benzene ring to avoid it coming into close proximity with the flavone core.

\section{Supramolecular features}

In the crystal, the title molecules form a parquet-like structure, with alternating layers of coplanar chromone backbones (Fig. 2). The presence of $\pi-\pi$ interactions in the crystal packing can be suggested from the short intermolecular distance of 3.299 (4) $\AA$ between the overlapping C9 atoms from opposing molecules. Moreover, a short contact of 3.286 (3) $\AA$ between the carbonyl oxygen atom and the centroid of the opposing heterocyclic ring is found, which suggests an interaction of the oxygen atom with the $\pi$-system (Fig. 3). Such an intermolecular interaction was found in the crystal structure of chiral amino alcohol with a pentafluorophenyl group (Korenaga et al., 2003). Two short $\mathrm{C}-\mathrm{H} \cdots \mathrm{O}$ contacts also occur, indicating at additional structural stability (Table 1).

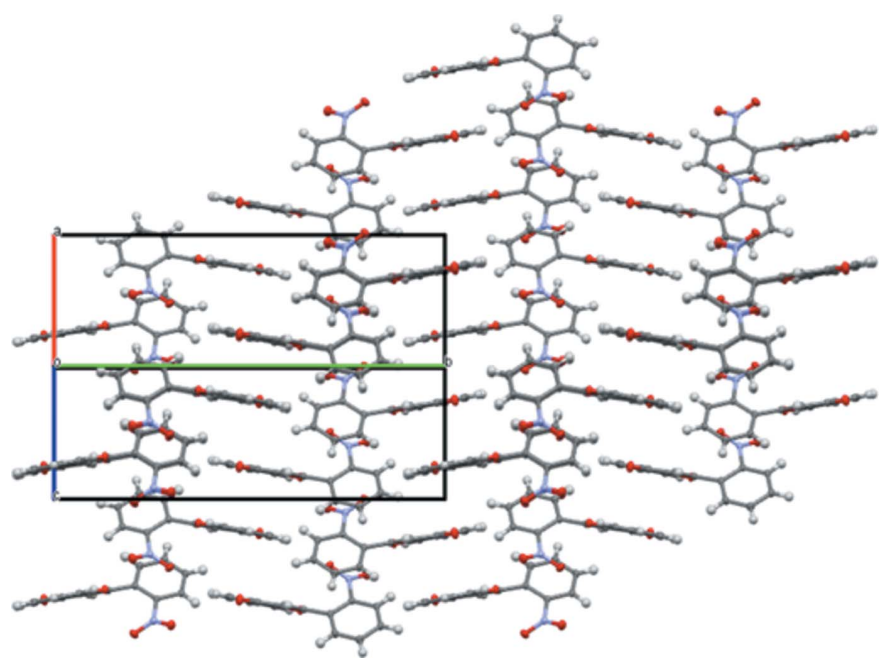

Figure 2

Parquet-like molecular packing in the title structure. 


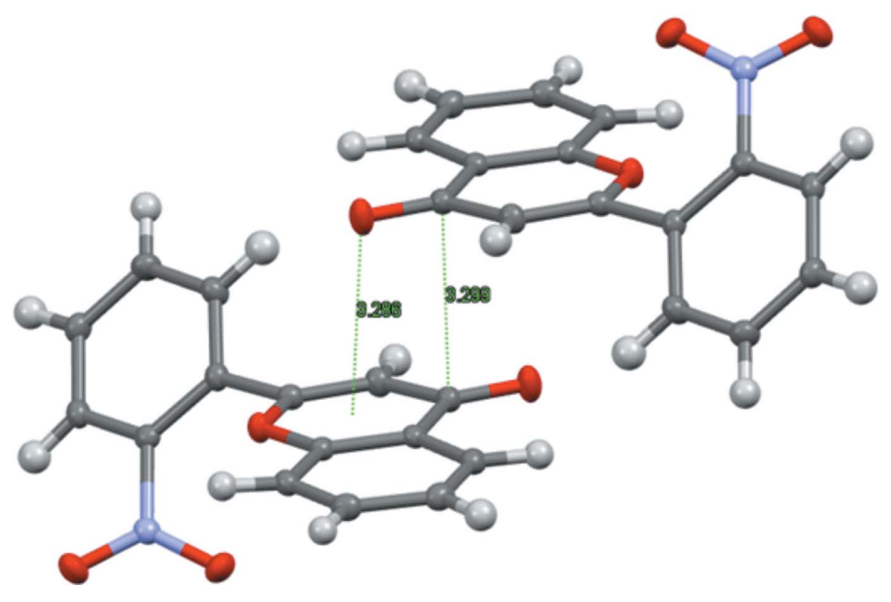

Figure 3

Short intermolecular $\mathrm{C} \cdots \mathrm{C}$ and $\mathrm{C}-\mathrm{O} \cdots \pi$ contacts in the crystal of the title compound.

\section{Database survey}

A search of the Cambridge Crystallographic Database (CSD version 5.40, update of September 19; Groom et al., 2016) for the title molecule yielded no entries. A single nitroflavone entry, for 2'-methyl-3'-nitroflavone, was found (REZROD; Kendi et al., 1996). A search for flavone-core molecules with only an ortho-substituted phenyl ring returned a total of six entries, three of which correspond to the compound with a methoxy group in the 2'-position [KEPLAS (Wallet et al., 1990), KEPLAS01 (McKendall et al., 2008), KEPLAS02 (Zia et al.., 2020)]; more specifically, one of the entries represents a structure of a possible polymorph, while the other two correspond to the same form. The other three correspond to carbazole (XIJVAQ; Zheng, 2018), hydroxy (YUDWEZ; Seetharaman \& Rajan, 1995) and ethyl glycolate (PIGXUB; Goyal et al., 2018) substituents. Most of these molecules

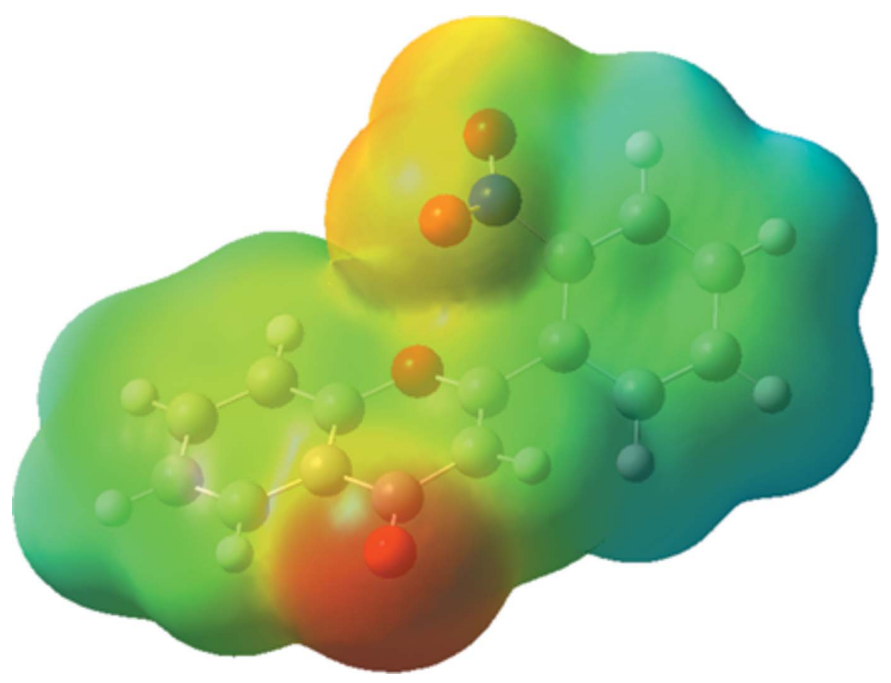

Figure 4

Electrostatic potential on the van der Waals surface of the title compound.
Table 2

Experimental (XRD) and calculated (DFT) dihedral angles $\left({ }^{\circ}\right)$ between the phenyl and chromone moieties in the title molecule (I), the $2^{\prime}$ methoxy derivative (II) and the $2^{\prime}$-carbazole derivative (III).

\begin{tabular}{lll}
\hline & XRD & DFT \\
\hline (I) & $50.73(5)$ & 47.56 \\
(II) & $2.89(7)$ & 22.16 \\
(III) & $29.21(6)$ & 40.13 \\
\hline
\end{tabular}

Table 3

Experimental (XRD) and calculated (DFT) lengths of single bonds $(\AA)$ between the phenyl and chromone moieties in the title molecule (I), the $2^{\prime}$-methoxy derivative (II) and the 2 -carbazole derivative (III).

\begin{tabular}{lll}
\hline & XRD & DFT \\
\hline (I) & $1.469(2)$ & 1.482 \\
(II) & $1.475(4)$ & 1.477 \\
(III) & $1.478(2)$ & 1.481 \\
\hline
\end{tabular}

Table 4

Experimental (XRD) and calculated (DFT) dihedral/torsion angles $\left(^{\circ}\right.$ ) between the phenyl group and the substituent in the $2^{\prime}$-position in the title molecule (I), the methoxy derivative (II) and the carbazole derivative (III).

\begin{tabular}{lll}
\hline & XRD & DFT \\
\hline (I) & $30.89(7)$ & 31.83 \\
(II) & $174.3(2)$ & 176.78 \\
(III) & $69.95(9)$ & 66.40 \\
\hline
\end{tabular}

exhibit only slight deviations from planarity, with the exception of carbazole-substituted molecule.

\section{DFT calculations}

In an attempt to get further insight into structure and properties of the title molecule (I), a DFT study was carried out at the B3LYP/6-31G* level of theory with GAUSSIAN 16 (Frisch et al., 2016) software. The geometry of the ground state was optimized, using the XRD data as a starting point. The optimized geometry was confirmed to be the minimum by vibrational frequency analysis. Two previously described flavonoids, with methoxy (II) and carbazole (III) substituents in the $2^{\prime}$-position, were also optimized and compared with the XRD data. Selected geometrical parameters are presented in Tables 2-4.

The calculated parameters are in satisfactory agreement with those obtained experimentally. The range of calculated dihedral angles between the moieties comprising the flavone core is narrower than that observed in the crystal structures, but still demonstrates the same trend with the title compound having the largest angle.

Considering the importance of the biological functions of the title compound, including its ability to competitively bind to benzodiazepine receptors, the electrostatic potential on the van der Waals surface was calculated (Fig. 4). While initially we had expected the nitro group to be the negative charge concentration site, it turned out to be the oxygen of the 
chromone moiety. We speculate that it could be the binding part in this molecule's interaction with benzodiazepine receptors.

\section{Synthesis and crystallization}

The synthesis of the title compound was performed as described in the literature (Barros \& Silva, 2006). The obtained product was recrystallized by slow evaporation from ethanol solution.

\section{Refinement}

Crystal data, data collection and structure refinement details are summarized in Table 5. Data collection was performed at $100 \mathrm{~K}$. All hydrogen atoms were located from the differenceFourier map and freely refined.

\section{Funding information}

Funding for this research was provided by: National Science Foundation (award No. DMR-0934212; award No. DMR1523611, PREM); National Institutes of Health (award No. 1R21NS084353-01).

\section{References}

Agati, G., Azzarello, E., Pollastri, S. \& Tattini, M. (2012). Plant Sci. 196, 67-76.

Barros, A. I. R. N. A. \& Silva, A. M. S. (2006). Monatsh. Chem. 137, 1505-1528.

Blank, V. C., Poli, C., Marder, M. \& Roguin, L. P. (2004). Bioorg. Med. Chem. Lett. 14, 133-136.

Bruker (2016). APEX3 and SAINT. Bruker AXS Inc., Madison, Wisconsin, USA.

Cárdenas, M. G., Blank, V. C., Marder, M. \& Roguin, L. P. (2008). Cancer Lett. 268, 146-157.

Cárdenas, M. G., Blank, V. C., Marder, M. N. \& Roguin, L. P. (2012). Anticancer Drugs, 23, 815-826.

Cárdenas, M. G., Zotta, E., Marder, M. \& Roguin, L. P. (2009). Int. J. Cancer, 125, 222-228.

Frisch, M. J., Trucks, G. W., Schlegel, H. B., Scuseria, G. E., Robb, M. A., Cheeseman, J. R., Montgomery, J. A. Jr, Vreven, T., Kudin, K. N., Burant, J. C., Millam, J. M., Iyengar, S. S., Tomasi, J., Barone, V., Mennucci, B., Cossi, M., Scalmani, G., Rega, N., Petersson, G. A., Nakatsuji, H., Hada, M., Ehara, M., Toyota, K., Fukuda, R., Hasegawa, J., Ishida, M., Nakajima, T., Honda, Y., Kitao, O., Nakai, H., Klene, M., Li, X., Knox, J. E., Hratchian, H. P., Cross, J. B., Bakken, V., Adamo, C., Jaramillo, J., Gomperts, R., Stratmann, R. E., Yazyev, O., Austin, A. J., Cammi, R., Pomelli, C., Ochterski, J. W., Ayala, P. Y., Morokuma, K., Voth, G. A., Salvador, P., Dannenberg, J. J., Zakrzewski, V. G., Dapprich, S., Daniels, A. D., Strain, M. C., Farkas, O., Malick, D. K., Rabuck, A. D., Raghavachari, K., Foresman, J. B., Ortiz, J. V., Cui, Q., Baboul, A. G., Clifford, S., Cioslowski, J., Stefanov, B. B., Liu, G., Liashenko, A., Piskorz, P., Komaromi, I., Martin, R. L., Fox, D. J., Keith, T., AlLaham, M. A., Peng, C. Y., Nanayakkara, A., Challacombe, M., Gill, P. M. W., Johnson, B., Chen, W., Wong, M. W., Gonzalez, C. \&
Table 5

Experimental details.

\begin{tabular}{|c|c|}
\hline \multicolumn{2}{|l|}{ Crystal data } \\
\hline Chemical formula & $\mathrm{C}_{15} \mathrm{H}_{9} \mathrm{NO}_{4}$ \\
\hline$M_{\mathrm{r}}$ & 267.23 \\
\hline Crystal system, space group & Monoclinic, $P 2_{1} / c$ \\
\hline Temperature (K) & 100 \\
\hline$a, b, c(\AA)$ & 8.079 (7), $20.134(17), 7.915$ (7) \\
\hline$\beta\left({ }^{\circ}\right)$ & $116.647(18)$ \\
\hline$V\left(\AA^{6}\right)$ & $1150.6(16)$ \\
\hline$Z$ & 4 \\
\hline Radiation type & Мo $K \alpha$ \\
\hline$\mu\left(\mathrm{mm}^{-1}\right)$ & 0.11 \\
\hline Crystal size $(\mathrm{mm})$ & $0.35 \times 0.28 \times 0.25$ \\
\hline \multicolumn{2}{|l|}{ Data collection } \\
\hline Diffractometer & Bruker APEXII CCD \\
\hline $\begin{array}{l}\text { No. of measured, independent and } \\
\text { observed }[I>2 \sigma(I)] \text { reflections }\end{array}$ & $9944,2491,2248$ \\
\hline$R_{\text {int }}$ & 0.034 \\
\hline$(\sin \theta / \lambda)_{\max }\left(\AA^{-1}\right)$ & 0.650 \\
\hline \multicolumn{2}{|l|}{ Refinement } \\
\hline$R\left[F^{2}>2 \sigma\left(F^{2}\right)\right], w R\left(F^{2}\right), S$ & $0.035,0.093,1.05$ \\
\hline No. of reflections & 2491 \\
\hline No. of parameters & 217 \\
\hline $\mathrm{H}$-atom treatment & All H-atom parameters refined \\
\hline$\Delta \rho_{\max }, \Delta \rho_{\min }\left(\mathrm{e} \AA^{-3}\right)$ & $0.30,-0.23$ \\
\hline
\end{tabular}

Computer programs: APEX3 and SAINT (Bruker, 2016), SHELXT2017/1 (Sheldrick, 2015a), SHELXL2017/1 (Sheldrick, 2015b) and Mercury (Macrae et al., 2020).

Pople, J. A. (2016). GAUSSIAN016. Rev. C.01. Gaussian Inc., Wallingford, CT, USA. http://www.gaussian.com.

Goyal, N., Do, C., Donahue, J. P., Mague, J. T. \& Foroozesh, M. (2018). IUCrData, 3, x180993.

Groom, C. R., Bruno, I. J., Lightfoot, M. P. \& Ward, S. C. (2016). Acta Cryst. B72, 171-179.

Kendi, E., Özbey, S., Tunçbilek, M. \& Ertan, R. (1996). Cryst. Res. Technol. 31, 611-615.

Korenaga, T., Tanaka, H., Ema, T. \& Sakai, T. (2003). J. Fluor. Chem. 122, 201-205.

Macrae, C. F., Sovago, I., Cottrell, S. J., Galek, P. T. A., McCabe, P., Pidcock, E., Platings, M., Shields, G. P., Stevens, J. S., Towler, M. \& Wood, P. A. (2020). J. Appl. Cryst. 53, 226-235.

Manthey, J., Grohmann, K. \& Guthrie, N. (2001). Curr. Med. Chem. 8, 135-153.

Marder, M., Viola, H., Wasowski, C., Wolfman, C., Waterman, P. G., Medina, J. H. \& Paladini, A. C. (1995). Bioorg. Med. Chem. Lett. 5, 2717-2720.

McKendall, M., Smith, T., Anh, K., Ellis, J., McGee, T., Foroozesh, M., Zhu, N. \& Stevens, C. L. K. (2008). J. Chem. Crystallogr. 38, 231237.

Ramos, S. (2008). Mol. Nutr. Food Res. 52, 507-526.

Seetharaman, J. \& Rajan, S. S. (1995). Z. Kristallogr. 210, 104-106.

Sheldrick, G. M. (2015a). Acta Cryst. A71, 3-8.

Sheldrick, G. M. (2015b). Acta Cryst. C71, 3-8.

Wallet, J.-C., Gaydou, E. M., Jaud, J. \& Baldy, A. (1990). Acta Cryst. C46, 1536-1540.

Zheng, Z. K. (2018). CSD Communication (refcode XIJVAQ). CCDC, Cambridge, England.

Zia, M., Khalid, M., Hameed, S., Irran, E. \& Naseer, M. M. (2020). J. Mol. Struct. 1207 Article 127811. 


\section{supporting information}

Acta Cryst. (2020). E76, 1417-1420 [https://doi.org/10.1107/S2056989020010713]

Structural (at $100 \mathrm{~K}$ ) and DFT studies of 2'-nitroflavone

Evgenii Oskolkov, Tatiana Kornilova, Preciosa America Chavez, John P. Tillotson and Tatiana V. Timofeeva

Computing details

Data collection: APEX3 (Bruker, 2016); cell refinement: SAINT (Bruker, 2016); data reduction: SAINT (Bruker, 2016); program(s) used to solve structure: SHELXT2017/1 (Sheldrick, 2015a); program(s) used to refine structure:

SHELXL2017/1 (Sheldrick, 2015b); molecular graphics: Mercury (Macrae et al., 2020); software used to prepare material for publication: Mercury (Macrae et al., 2020).

2-(2-Nitrophenyl)-4H-chromen-4-one

Crystal data

$\mathrm{C}_{15} \mathrm{H}_{9} \mathrm{NO}_{4}$

$M_{r}=267.23$

Monoclinic, $P 2{ }_{1} / c$

$a=8.079(7) \AA$

$b=20.134$ (17) $\AA$

$c=7.915(7) \AA$

$\beta=116.647(18)^{\circ}$

$V=1150.6(16) \AA^{3}$

$Z=4$

Data collection

Bruker APEXII CCD

diffractometer

$\varphi$ and $\omega$ scans

9944 measured reflections

2491 independent reflections

2248 reflections with $I>2 \sigma(I)$

Refinement

Refinement on $F^{2}$

Least-squares matrix: full

$R\left[F^{2}>2 \sigma\left(F^{2}\right)\right]=0.035$

$w R\left(F^{2}\right)=0.093$

$S=1.05$

2491 reflections

217 parameters

0 restraints
$F(000)=552$

$D_{\mathrm{x}}=1.543 \mathrm{Mg} \mathrm{m}^{-3}$

Mo $K \alpha$ radiation, $\lambda=0.71073 \AA$

Cell parameters from 1326 reflections

$\theta=3.1-32.1^{\circ}$

$\mu=0.11 \mathrm{~mm}^{-1}$

$T=100 \mathrm{~K}$

Block-shaped, white

$0.35 \times 0.28 \times 0.25 \mathrm{~mm}$

$R_{\text {int }}=0.034$

$\theta_{\max }=27.5^{\circ}, \theta_{\min }=2.0^{\circ}$

$h=-10 \rightarrow 6$

$k=-16 \rightarrow 26$

$l=-10 \rightarrow 8$

Primary atom site location: iterative

Hydrogen site location: difference Fourier map

All $\mathrm{H}$-atom parameters refined

$w=1 /\left[\sigma^{2}\left(F_{\mathrm{o}}^{2}\right)+(0.0401 P)^{2}+0.551 P\right]$

where $P=\left(F_{\mathrm{o}}^{2}+2 F_{\mathrm{c}}^{2}\right) / 3$

$(\Delta / \sigma)_{\max }=0.001$

$\Delta \rho_{\max }=0.30 \mathrm{e} \AA^{-3}$

$\Delta \rho_{\min }=-0.23$ e $\AA^{-3}$ 


\section{Special details}

Geometry. All esds (except the esd in the dihedral angle between two 1.s. planes) are estimated using the full covariance matrix. The cell esds are taken into account individually in the estimation of esds in distances, angles and torsion angles; correlations between esds in cell parameters are only used when they are defined by crystal symmetry. An approximate (isotropic) treatment of cell esds is used for estimating esds involving l.s. planes.

Fractional atomic coordinates and isotropic or equivalent isotropic displacement parameters $\left(\AA^{2}\right)$

\begin{tabular}{|c|c|c|c|c|}
\hline & $x$ & $y$ & $z$ & $U_{\text {iso }} * / U_{\text {eq }}$ \\
\hline $\mathrm{O} 3$ & $0.22049(12)$ & $0.13047(4)$ & $0.89731(12)$ & $0.0160(2)$ \\
\hline $\mathrm{O} 4$ & $0.48862(13)$ & $-0.02543(4)$ & $1.23925(13)$ & $0.0229(2)$ \\
\hline $\mathrm{O} 1$ & $0.04077(13)$ & $0.29953(4)$ & $0.98796(14)$ & $0.0230(2)$ \\
\hline $\mathrm{O} 2$ & $0.11312(14)$ & $0.20433(4)$ & $1.12606(14)$ & $0.0243(2)$ \\
\hline $\mathrm{C} 7$ & $0.37958(17)$ & $0.13828(6)$ & $1.06058(17)$ & $0.0154(2)$ \\
\hline $\mathrm{C} 10$ & $0.22802(17)$ & $0.01385(6)$ & $0.96633(16)$ & $0.0146(2)$ \\
\hline C9 & $0.40481(17)$ & $0.02123(6)$ & $1.13800(17)$ & $0.0160(2)$ \\
\hline $\mathrm{C} 15$ & $0.14347(17)$ & $0.06830(5)$ & $0.85277(17)$ & $0.0144(2)$ \\
\hline C14 & $-0.02257(17)$ & $0.06319(6)$ & $0.68804(17)$ & $0.0163(2)$ \\
\hline $\mathrm{C} 2$ & $0.33977(17)$ & $0.26206(6)$ & $1.08494(16)$ & $0.0148(2)$ \\
\hline $\mathrm{C} 13$ & $-0.10846(18)$ & $0.00246(6)$ & $0.63791(17)$ & $0.0182(3)$ \\
\hline $\mathrm{C} 1$ & $0.44677(17)$ & $0.20715(6)$ & $1.09092(16)$ & $0.0154(2)$ \\
\hline N1 & $0.15066(15)$ & $0.25441(5)$ & $1.06335(14)$ & $0.0164(2)$ \\
\hline $\mathrm{C} 8$ & $0.47167(18)$ & $0.08861(6)$ & 1.17707 (17) & $0.0175(3)$ \\
\hline C11 & $0.13820(18)$ & $-0.04727(6)$ & $0.91170(17)$ & $0.0168(3)$ \\
\hline C6 & $0.62821(18)$ & $0.21870(6)$ & $1.12309(18)$ & $0.0194(3)$ \\
\hline $\mathrm{C} 3$ & $0.40847(18)$ & $0.32564(6)$ & $1.10911(17)$ & $0.0165(3)$ \\
\hline $\mathrm{C} 5$ & 0.69942 (19) & $0.28224(6)$ & $1.14845(19)$ & $0.0204(3)$ \\
\hline $\mathrm{C} 4$ & $0.58971(18)$ & $0.33551(6)$ & $1.14135(17)$ & $0.0185(3)$ \\
\hline $\mathrm{C} 12$ & $-0.02751(18)$ & $-0.05292(6)$ & $0.75091(18)$ & $0.0186(3)$ \\
\hline H13 & $-0.221(2)$ & $-0.0009(7)$ & $0.527(2)$ & $0.019(4)^{*}$ \\
\hline H11 & $0.196(2)$ & $-0.0854(7)$ & $0.994(2)$ & $0.020(4)^{*}$ \\
\hline H8 & $0.585(2)$ & $0.0986(8)$ & $1.292(2)$ & $0.024(4)^{*}$ \\
\hline H14 & $-0.071(2)$ & $0.1017(8)$ & $0.610(2)$ & $0.020(4)^{*}$ \\
\hline $\mathrm{H} 3$ & $0.334(2)$ & $0.3611(8)$ & $1.110(2)$ & $0.025(4)^{*}$ \\
\hline $\mathrm{H} 4$ & $0.638(2)$ & $0.3804(8)$ & $1.162(2)$ & $0.025(4)^{*}$ \\
\hline H5 & $0.825(2)$ & $0.2894(7)$ & $1.172(2)$ & $0.022(4)^{*}$ \\
\hline H12 & $-0.090(2)$ & $-0.0948(8)$ & $0.716(2)$ & $0.024(4)^{*}$ \\
\hline H6 & $0.706(2)$ & $0.1816(8)$ & $1.128(2)$ & $0.027(4)^{*}$ \\
\hline
\end{tabular}

Atomic displacement parameters $\left(\AA^{2}\right)$

\begin{tabular}{lllllll}
\hline & $U^{11}$ & $U^{22}$ & $U^{33}$ & $U^{12}$ & $U^{13}$ & $U^{23}$ \\
\hline O3 & $0.0160(5)$ & $0.0119(4)$ & $0.0168(4)$ & $-0.0011(3)$ & $0.0043(4)$ & $-0.0001(3)$ \\
O4 & $0.0205(5)$ & $0.0164(4)$ & $0.0244(5)$ & $0.0012(3)$ & $0.0034(4)$ & $0.0050(3)$ \\
O1 & $0.0174(5)$ & $0.0199(4)$ & $0.0291(5)$ & $0.0041(3)$ & $0.0083(4)$ & $0.0014(4)$ \\
O2 & $0.0265(5)$ & $0.0205(4)$ & $0.0309(5)$ & $-0.0026(4)$ & $0.0175(5)$ & $0.0039(4)$ \\
C7 & $0.0149(6)$ & $0.0148(5)$ & $0.0168(5)$ & $-0.0012(4)$ & $0.0073(5)$ & $-0.0026(4)$ \\
C10 & $0.0151(6)$ & $0.0138(5)$ & $0.0166(5)$ & $0.0006(4)$ & $0.0086(5)$ & $-0.0012(4)$
\end{tabular}




\begin{tabular}{lllllll} 
C9 & $0.0158(6)$ & $0.0151(5)$ & $0.0173(5)$ & $0.0008(4)$ & $0.0077(5)$ & $0.0007(4)$ \\
C15 & $0.0161(6)$ & $0.0125(5)$ & $0.0169(5)$ & $-0.0010(4)$ & $0.0094(5)$ & $-0.0021(4)$ \\
C14 & $0.0164(6)$ & $0.0163(5)$ & $0.0164(5)$ & $0.0021(4)$ & $0.0076(5)$ & $0.0005(4)$ \\
C2 & $0.0150(6)$ & $0.0162(5)$ & $0.0132(5)$ & $-0.0006(4)$ & $0.0062(5)$ & $-0.0005(4)$ \\
C13 & $0.0158(6)$ & $0.0215(6)$ & $0.0159(5)$ & $-0.0023(5)$ & $0.0059(5)$ & $-0.0034(4)$ \\
C1 & $0.0173(6)$ & $0.0129(5)$ & $0.0146(5)$ & $-0.0005(4)$ & $0.0059(5)$ & $-0.0006(4)$ \\
N1 & $0.0174(5)$ & $0.0156(5)$ & $0.0162(5)$ & $0.0000(4)$ & $0.0076(4)$ & $-0.0020(4)$ \\
C8 & $0.0165(6)$ & $0.0160(6)$ & $0.0169(5)$ & $-0.0005(4)$ & $0.0046(5)$ & $-0.0014(4)$ \\
C11 & $0.0191(6)$ & $0.0139(5)$ & $0.0195(6)$ & $0.0006(4)$ & $0.0106(5)$ & $-0.0009(4)$ \\
C6 & $0.0182(7)$ & $0.0156(6)$ & $0.0236(6)$ & $0.0009(5)$ & $0.0087(5)$ & $-0.0017(5)$ \\
C3 & $0.0201(6)$ & $0.0130(5)$ & $0.0161(5)$ & $0.0010(4)$ & $0.0078(5)$ & $0.0000(4)$ \\
C5 & $0.0163(7)$ & $0.0195(6)$ & $0.0252(6)$ & $-0.0023(5)$ & $0.0092(5)$ & $-0.0012(5)$ \\
C4 & $0.0211(7)$ & $0.0142(5)$ & $0.0201(6)$ & $-0.0032(5)$ & $0.0091(5)$ & $-0.0012(4)$ \\
C12 & $0.0215(7)$ & $0.0157(5)$ & $0.0209(6)$ & $-0.0037(5)$ & $0.0115(5)$ & $-0.0043(4)$ \\
\hline
\end{tabular}

Geometric parameters $\left(\AA,{ }^{\circ}\right)$

\begin{tabular}{|c|c|c|c|}
\hline $\mathrm{O} 3-\mathrm{C} 7$ & $1.3626(17)$ & $\mathrm{C} 2-\mathrm{C} 3$ & $1.3741(19)$ \\
\hline $\mathrm{O} 3-\mathrm{C} 15$ & $1.3717(16)$ & $\mathrm{C} 13-\mathrm{C} 12$ & $1.3950(19)$ \\
\hline $\mathrm{O} 4-\mathrm{C} 9$ & $1.2233(16)$ & $\mathrm{C} 1-\mathrm{C} 6$ & $1.390(2)$ \\
\hline $\mathrm{O} 1-\mathrm{N} 1$ & $1.2224(15)$ & $\mathrm{C} 11-\mathrm{C} 12$ & $1.377(2)$ \\
\hline $\mathrm{O} 2-\mathrm{N} 1$ & $1.2208(15)$ & $\mathrm{C} 6-\mathrm{C} 5$ & $1.380(2)$ \\
\hline $\mathrm{C} 7-\mathrm{C} 1$ & $1.4690(19)$ & $\mathrm{C} 3-\mathrm{C} 4$ & $1.383(2)$ \\
\hline $\mathrm{C} 7-\mathrm{C} 8$ & $1.3373(18)$ & $\mathrm{C} 5-\mathrm{C} 4$ & 1.3765 (19) \\
\hline $\mathrm{C} 10-\mathrm{C} 9$ & $1.4722(19)$ & $\mathrm{C} 14-\mathrm{H} 14$ & $0.958(15)$ \\
\hline $\mathrm{C} 10-\mathrm{C} 15$ & $1.3877(18)$ & $\mathrm{C} 13-\mathrm{H} 13$ & $0.944(16)$ \\
\hline $\mathrm{C} 10-\mathrm{C} 11$ & $1.3950(19)$ & $\mathrm{C} 11-\mathrm{H} 11$ & $0.979(15)$ \\
\hline $\mathrm{C} 9-\mathrm{C} 8$ & 1.4415 (19) & $\mathrm{C} 6-\mathrm{H} 6$ & 0.964 (17) \\
\hline $\mathrm{C} 15-\mathrm{C} 14$ & $1.3932(19)$ & $\mathrm{C} 3-\mathrm{H} 3$ & $0.933(17)$ \\
\hline $\mathrm{C} 14-\mathrm{C} 13$ & $1.3736(19)$ & $\mathrm{C} 5-\mathrm{H} 5$ & $0.953(17)$ \\
\hline $\mathrm{C} 2-\mathrm{C} 1$ & $1.3911(18)$ & $\mathrm{C} 4-\mathrm{H} 4$ & $0.971(16)$ \\
\hline $\mathrm{C} 2-\mathrm{N} 1$ & $1.467(2)$ & $\mathrm{C} 12-\mathrm{H} 12$ & $0.957(16)$ \\
\hline $\mathrm{C} 7-\mathrm{O} 3-\mathrm{C} 15$ & $118.38(9)$ & $\mathrm{C} 12-\mathrm{C} 11-\mathrm{C} 10$ & $120.95(12)$ \\
\hline $\mathrm{O} 3-\mathrm{C} 7-\mathrm{C} 1$ & $112.72(10)$ & $\mathrm{C} 5-\mathrm{C} 6-\mathrm{C} 1$ & $121.21(12)$ \\
\hline $\mathrm{C} 8-\mathrm{C} 7-\mathrm{O} 3$ & 124.33 (11) & $\mathrm{C} 2-\mathrm{C} 3-\mathrm{C} 4$ & $119.18(11)$ \\
\hline $\mathrm{C} 8-\mathrm{C} 7-\mathrm{C} 1$ & $122.86(12)$ & $\mathrm{C} 4-\mathrm{C} 5-\mathrm{C} 6$ & $119.85(14)$ \\
\hline $\mathrm{C} 15-\mathrm{C} 10-\mathrm{C} 9$ & $120.66(11)$ & $\mathrm{C} 5-\mathrm{C} 4-\mathrm{C} 3$ & $120.30(12)$ \\
\hline $\mathrm{C} 15-\mathrm{C} 10-\mathrm{C} 11$ & $117.36(12)$ & $\mathrm{C} 11-\mathrm{C} 12-\mathrm{C} 13$ & $120.55(12)$ \\
\hline $\mathrm{C} 11-\mathrm{C} 10-\mathrm{C} 9$ & $121.98(11)$ & $\mathrm{C} 13-\mathrm{C} 14-\mathrm{H} 14$ & $121.7(9)$ \\
\hline $\mathrm{O} 4-\mathrm{C} 9-\mathrm{C} 10$ & $123.36(12)$ & $\mathrm{C} 15-\mathrm{C} 14-\mathrm{H} 14$ & $119.2(9)$ \\
\hline $\mathrm{O} 4-\mathrm{C} 9-\mathrm{C} 8$ & $122.56(13)$ & $\mathrm{C} 14-\mathrm{C} 13-\mathrm{H} 13$ & $119.0(9)$ \\
\hline $\mathrm{C} 8-\mathrm{C} 9-\mathrm{C} 10$ & $114.08(10)$ & $\mathrm{C} 12-\mathrm{C} 13-\mathrm{H} 13$ & $121.3(9)$ \\
\hline $\mathrm{O} 3-\mathrm{C} 15-\mathrm{C} 10$ & $121.31(12)$ & $\mathrm{C} 7-\mathrm{C} 8-\mathrm{H} 8$ & $119.2(9)$ \\
\hline $\mathrm{O} 3-\mathrm{C} 15-\mathrm{C} 14$ & $116.32(10)$ & $\mathrm{C} 9-\mathrm{C} 8-\mathrm{H} 8$ & $119.7(9)$ \\
\hline $\mathrm{C} 10-\mathrm{C} 15-\mathrm{C} 14$ & $122.37(11)$ & $\mathrm{C} 12-\mathrm{C} 11-\mathrm{H} 11$ & $121.7(9)$ \\
\hline $\mathrm{C} 13-\mathrm{C} 14-\mathrm{C} 15$ & $119.06(11)$ & $\mathrm{C} 10-\mathrm{C} 11-\mathrm{H} 11$ & $117.3(9)$ \\
\hline $\mathrm{C} 1-\mathrm{C} 2-\mathrm{N} 1$ & $121.28(11)$ & $\mathrm{C} 5-\mathrm{C} 6-\mathrm{H} 6$ & $119.4(10)$ \\
\hline
\end{tabular}




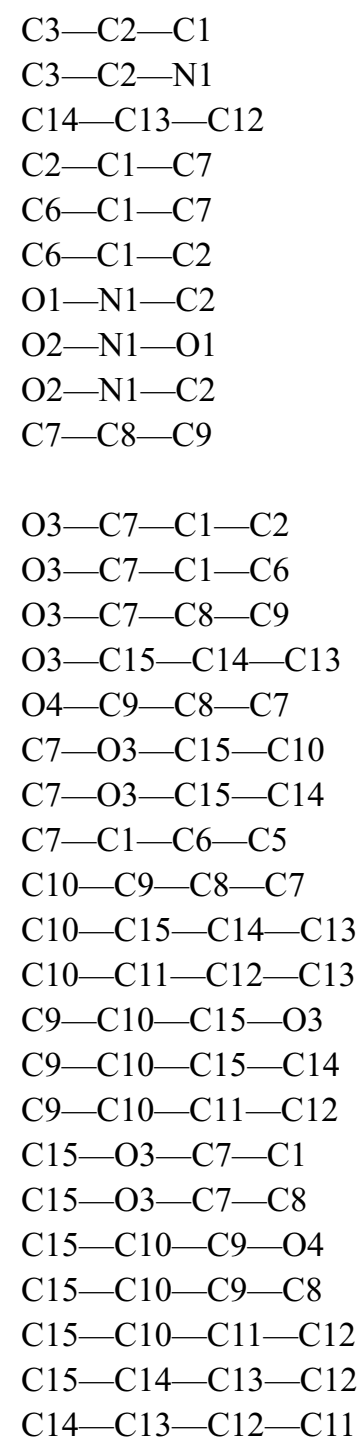

$121.98(13)$

$116.64(11)$

$119.70(13)$

$124.49(12)$

$118.02(11)$

$117.47(11)$

$117.98(11)$

$123.63(12)$

$118.35(10)$

$121.12(12)$

$52.15(16)$

$-126.13(13)$

$-1.1(2)$

$-178.69(11)$

$178.00(12)$

-2.47 (17)

$177.77(10)$

$178.51(12)$

$-1.92(18)$

1.55 (19)

$0.61(19)$

-0.57 (18)

$179.18(11)$

$179.73(11)$

$-179.96(10)$

3.39 (18)

$-177.23(12)$

$2.69(17)$

$0.18(18)$

-0.71 (19)

-0.33 (19)
$\mathrm{C} 1-\mathrm{C} 6-\mathrm{H} 6$

$\mathrm{C} 2-\mathrm{C} 3-\mathrm{H} 3$

$\mathrm{C} 4-\mathrm{C} 3-\mathrm{H} 3$

$\mathrm{C} 4-\mathrm{C} 5-\mathrm{H} 5$

$\mathrm{C} 6-\mathrm{C} 5-\mathrm{H} 5$

$\mathrm{C} 5-\mathrm{C} 4-\mathrm{H} 4$

$\mathrm{C} 3-\mathrm{C} 4-\mathrm{H} 4$

$\mathrm{C} 11-\mathrm{C} 12-\mathrm{H} 12$

C13-C12-H12

$\mathrm{C} 2-\mathrm{C} 1-\mathrm{C} 6-\mathrm{C} 5$

$\mathrm{C} 2-\mathrm{C} 3-\mathrm{C} 4-\mathrm{C} 5$

$\mathrm{C} 1-\mathrm{C} 7-\mathrm{C} 8-\mathrm{C} 9$

$\mathrm{C} 1-\mathrm{C} 2-\mathrm{N} 1-\mathrm{O} 1$

$\mathrm{C} 1-\mathrm{C} 2-\mathrm{N} 1-\mathrm{O} 2$

$\mathrm{C} 1-\mathrm{C} 2-\mathrm{C} 3-\mathrm{C} 4$

$\mathrm{C} 1-\mathrm{C} 6-\mathrm{C} 5-\mathrm{C} 4$

$\mathrm{N} 1-\mathrm{C} 2-\mathrm{C} 1-\mathrm{C} 7$

$\mathrm{N} 1-\mathrm{C} 2-\mathrm{C} 1-\mathrm{C} 6$

$\mathrm{N} 1-\mathrm{C} 2-\mathrm{C} 3-\mathrm{C} 4$

$\mathrm{C} 8-\mathrm{C} 7-\mathrm{C} 1-\mathrm{C} 2$

$\mathrm{C} 8-\mathrm{C} 7-\mathrm{C} 1-\mathrm{C} 6$

$\mathrm{C} 11-\mathrm{C} 10-\mathrm{C} 9-\mathrm{O} 4$

$\mathrm{C} 11-\mathrm{C} 10-\mathrm{C} 9-\mathrm{C} 8$

$\mathrm{C} 11-\mathrm{C} 10-\mathrm{C} 15-\mathrm{O} 3$

$\mathrm{C} 11-\mathrm{C} 10-\mathrm{C} 15-\mathrm{C} 14$

$\mathrm{C} 6-\mathrm{C} 5-\mathrm{C} 4-\mathrm{C} 3$

$\mathrm{C} 3-\mathrm{C} 2-\mathrm{C} 1-\mathrm{C} 7$

$\mathrm{C} 3-\mathrm{C} 2-\mathrm{C} 1-\mathrm{C} 6$

$\mathrm{C} 3-\mathrm{C} 2-\mathrm{N} 1-\mathrm{O} 1$

$\mathrm{C} 3-\mathrm{C} 2-\mathrm{N} 1-\mathrm{O} 2$
$119.4(10)$

$119.6(10)$

$121.1(10)$

$119.8(9)$

$120.4(9)$

120.9 (10)

$118.8(10)$

$120.5(9)$

$118.9(9)$

$0.11(19)$

$0.21(18)$

$-177.40(11)$

$-152.23(12)$

29.74 (16)

-0.34 (18)

$-0.2(2)$

$5.50(17)$

$-176.22(10)$

$176.21(10)$

$-131.15(14)$

50.57 (18)

$3.2(2)$

$-176.84(11)$

$178.98(11)$

$-1.27(18)$

0.07 (19)

$-178.11(11)$

$0.18(18)$

$31.19(15)$

$-146.84(12)$

Hydrogen-bond geometry $\left(\AA,{ }^{\circ}\right)$

\begin{tabular}{lllll}
\hline$D-\mathrm{H} \cdots A$ & $D-\mathrm{H}$ & $\mathrm{H} \cdots A$ & $D \cdots A$ & $D-\mathrm{H} \cdots A$ \\
\hline $\mathrm{C} 3-\mathrm{H} 3 \cdots \mathrm{O} 44^{\mathrm{i}}$ & $0.933(17)$ & $2.675(16)$ & $3.198(3)$ & $116.1(12)$ \\
$\mathrm{C} 4-\mathrm{H} 4 \cdots \mathrm{O} 4{ }^{\mathrm{i}}$ & $0.971(16)$ & $2.446(16)$ & $3.109(3)$ & $125.3(12)$ \\
\hline
\end{tabular}

Symmetry code: (i) $-x+1, y+1 / 2,-z+5 / 2$. 